\title{
The Dynamics of Neuronal Death: A Time-Lapse Study in the Retina
}

\author{
Alessandro Cellerino, Lucia Galli-Resta, and Laura Colombaioni \\ Istituto di Neurofisiologia del Consiglio Nazionale delle Ricerche, 56017 Pisa, Italy
}

Using time-lapse video microscopy, we have followed the process of neuronal death in an intact region of the mammalian nervous system. We show here the fast dynamics of nuclear fragmentation, which is over in $<1 \mathrm{hr}$ for neurons undergoing apoptosis in the living rat retina. Nuclear fragmentation is ac- companied by a progressive raise of intracellular calcium and followed by erratic movement of the apoptotic cells, documenting their loss of adhesion.

Key words: apoptosis; rat; calcium; nuclear fragmentation; pyknosis; Fluo-3
Programmed cellular death (PCD) is essential for cell number homeostasis in the organism, is widespread during nervous system development, and is involved in several neurodegenerative diseases. Since the pioneering studies of Montalcini and Hamburger (1949), much has been learned about PCD in the nervous system, including its exact developmental profile and its extent in a large number of peripheral and central neuronal populations (Oppenheim, 1981, 1991). The elucidation of the molecular mechanisms involved in apoptosis is proceeding at an ever increasing rate (Pettmann and Henderson, 1998), and pharmacological tools aimed at interfering with apoptosis induction are being developed (Schulz et al., 1999). Yet, considerable basic information is still missing about the cellular dynamics of the death process in the nervous system. The exact progression of nuclear and cytoplasmic alterations has been only inferred from histological studies, and even the time required for the execution of the death program is still unknown. These and related questions can be answered by a time-lapse study in which single cells are followed throughout the apoptotic process. Until now, this approach was attempted in dissociated cells and immortalized cell lines (Pittman et al., 1993; MacManus et al., 1997; Messam and Pittman, 1998), but it is unclear to what extent the notions thus acquired apply to neurons dying in vivo.

In the present study, we followed the dynamic aspects of apoptosis in an intact region of the mammalian CNS, the neonatal rat retina, which can be isolated in its integrity and cultivated in vitro (Linden et al., 1999). In this model system, we could follow neonatal retinal ganglion cells (RGCs) undergoing apoptosis, as they normally do in vivo at these ages. Using time-lapse video recording, cell labeling, and a fluorescent $\mathrm{Ca}^{2+}$ indicator, we determined the dynamics of nuclear condensation in RGCs undergoing apoptosis, the time course of this process, the parallel rise of intracellular $\mathrm{Ca}^{2+}$, and the loss of cell adhesion accompanying cell death.

\section{MATERIALS AND METHODS}

Experiments were performed with Long-Evans hooded rats in compliance with the guidelines of the Association for Research in Vision and Ophthalmology (ARVO) for the use of experimental animals.

Retrograde labeling. RGCs were labeled with the retrograde nuclear

Received Feb. 23, 2000; revised May 8, 2000; accepted June 14, 2000.

This work was supported by the Consiglio Nazionale delle Ricerche and the European Commission, DGXII, Biotechnology Program. We thank Giulio Cappagli for technical assistance.

Correspondence should be addressed to Dr. Laura Colombaioni, Istituto di Neurofisiologia del Consiglio Nazionale delle Ricerche, via G. Moruzzi 1, 56100 Pisa, Italy. E-mail: colomb@in.pi.cnr.it.

Copyright (C) 2000 Society for Neuroscience $\quad 0270-6474 / 00 / 200001-05 \$ 15.00 / 0$ tracer Diamidino Yellow (DiY, Sigma, St. Louis, MO). At postnatal day 0 (P0), newborn rats were anesthetized by hypothermia, the superior colliculus was visualized by trans-illumination, and 1-2 $\mu \mathrm{l}$ of DiY suspension $(1 \% \mathrm{w} / \mathrm{v})$ in PBS was injected through the skin and the skull cartilage using a glass microelectrode connected to a $10 \mu$ l Hamilton syringe. The pups were then revived under a table light and returned to their mother (Harvey and Robertson, 1992).

Retinal explantation, culture, and Fluo-3-AM loading. Retinas were explanted on $\mathrm{P} 0-\mathrm{P} 2$ after the animals were decapitated. Eyes were removed and opened by an encircling cut, and the cornea and lens were removed. The retina was separated from the pigment epithelium, and RGCs were placed facing down in a chambered coverglass (Lab-Tek, Nunc, Naperville, IL) filled with MEM, pH 7.4. The incubation chamber was placed within a Plexiglas incubator (Zeiss, model M 411851) fitted on the heated stage of an inverted microscope (Zeiss, Axiovert 135). A temperature controller (Zeiss, 37-2) and a $\mathrm{CO}_{2}$ controller (Zeiss CTI 3700 ) maintained the retina at $37^{\circ} \mathrm{C}$ in $5 \% \mathrm{CO}_{2} / 95 \% \mathrm{O}_{2}$. Retinas were left to stabilize $7-8 \mathrm{hr}$ after explantation, before the imaging session was started. To perform $\mathrm{Ca}^{2+}$ imaging, retinas were incubated for $1 \mathrm{hr}$ at $37^{\circ} \mathrm{C}$ in a $2 \mu \mathrm{M}$ solution of Fluo-3-AM in MEM with $0.2 \%$ DMSO, followed by several washes in MEM, before the imaging session.

Imaging. Images were acquired by means of a CCD cooled camera (KAF-400E, DTA, Pisa, Italy) connected to the microscope and driven by a dedicated software. The intensity of the excitation source reaching the tissue was dimmed using an Attoarc (Zeiss). An electronically driven shutter allowed us to expose the preparation to the excitation light only while images were taken. DiY fluorescence images were acquired using a UV filter (Zeiss, BP 365, LP 397), and Fluo-3 fluorescence was acquired using a fluorescein filter (Zeiss, BP 450-490, LP 520). Images of the same field $\left(175 \times 115 \mu \mathrm{m}^{2}\right)$ of the retinal ganglion cell layer were taken at regular time intervals using a $40 \times$ immersion objective, leading to a final image resolution of $0.22 \mu \mathrm{m} /$ pixel. Exposure times were 10-20 $\mathrm{msec}$ for DiY and 0.5-2 sec for Fluo-3. With these imaging conditions, single fields were routinely monitored for $2-3 \mathrm{hr}$ without any indication of photo damage.

Fluorescence quantification. The images acquired were exported to an image analyzer (MCID, Imaging research Inc., Ontario, Canada) to quantify fluorescence levels within cells showing an increase of Fluo-3 fluorescence emission. Within each frame, the fluorescence intensity emitted by a cell was determined as the average gray level over the cell somata, normalized to the background level. Each cell analyzed was

This article is published in The Journal of Neuroscience, Rapid Communications Section, which publishes brief, peer-reviewed papers online, not in print. Rapid Communications are posted online approximately one month earlier than they would appear if printed. They are listed in the Table of Contents of the next open issue of JNeurosci. Cite this article as: JNeurosci, 2000, 20:RC92 (1-5). The publication date is the date of posting online at www.jneurosci.org.

http://www.jneurosci.org/cgi/content/full/4466 
completely covered by nonoverlapping sampling squares $5 \times 5$ pixel $^{2}$ in size. The mean of the gray values determined in all the squares entirely contained within the cell was defined as the gray value of the cell $\left(F_{\text {cell }}\right)$. This allowed us to avoid the subjective errors determined by manually outlining a cell profile. The gray value averaged over a fixed 2500 pixel $^{2}$ region devoid of fluorescent cells was taken as the background gray value $\left(F_{\text {background }}\right)$. The ratio $F_{\text {cell }} / F_{\text {background }}$ was taken as a measure of the fluorescence of the cell in the frame. To allow the comparison between different cells, the fluorescence of each cell was expressed as percentage of its maximal value. The time course of fluorescence intensity within each cell resulted in roughly sigmoidal curves that were superimposed on one another by aligning the midpoints. We limited our observations to a semiquantitative analysis of relative fluorescent emission increase and made no attempts to quantify the $\mathrm{Ca}^{2+}$ concentration corresponding to the fluorescent emission levels.

Analysis of cell movement. The coordinates of each moving cell were recorded in each frame, as the position of the center of the cell somata. The coordinates of stationary cells and other reference points were also acquired and used to correct for potential drift of the sampled field from frame to frame. After this correction, the vector connecting the positions taken by a cell in two consecutive frames was taken to estimate the cell displacement within the interval between two consecutive images. A minimal estimate of the total cell displacement during the recording session was obtained as the sum of the lengths of the displacements observed between consecutive frames.

\section{RESULTS}

\section{Dying cells in the living explanted retina}

The distinctive feature of neurons dying by programmed cell death is a pyknotic nucleus, where clumps of chromatin betray a massive DNA fragmentation (Wyllie et al., 1980). To follow the stages of nuclear alteration leading to overt nuclear fragmentation in RGCs, we have used the nuclear dye DiY, which was injected into the superior colliculus of neonatal rats $24-48 \mathrm{hr}$ before the experiment. Retrogradely labeled retinas were explanted and kept within an incubation chamber placed on the stage of an inverted microscope, to image living RGCs under controlled temperature and $\mathrm{CO}_{2}$ concentration. When viewed under epifluorescence, normal cell nuclei labeled with DiY showed a faint uniform fluorescence, whereas apoptotic RGCs were identified by the pyknotic appearance of their bright nucleus. In a first set of experiments, we monitored by time-lapse analysis single retinal fields to follow healthy looking RGCs until they completed the transition to pyknosis. Repetitive exposure to UV light, necessary to excite DiY, can rapidly induce photo damage, as betrayed by the almost simultaneous ballooning and nuclear rounding of most cells in the field (data not shown). This can be easily distinguished from apoptosis, which involves only scattered cells, and is characterized by a typical fragmentation of the nucleus (Wyllie et al., 1980). To minimize the risk of photo damage, we reduced the intensity of the UV light and the exposure times to a minimum and took images of the field every $3 \mathrm{~min}$. This allowed us to monitor each retinal field for 2-3 hr without any indication of photo damage.

\section{The dynamic of nuclear condensation in dying cells}

Apoptosis is a highly asynchronous process in the neonatal retina (Perry et al., 1983), and we could only follow zero to four RGCs undergoing apoptosis within the single retinal patch of $175 \times 115$ $\mu \mathrm{m}^{2}$ monitored in each recording session. The complete execution of nuclear fragmentation was followed for 15 RGCs in six successful experiments. Two examples are shown in Figure $1 A, B$. The number on each panel indicates the minutes since the beginning of the sequence. The duration of the whole process was remarkably consistent between different cells, requiring $40 \pm 3$ min (range 36-45 min) to complete. Some variations in the process of nuclear condensation were observed. In some cells (Fig. $1 A$ ), this started with the formation of many small clumps within the nucleus (Fig. 1A) (6-12 min); these clumps then condensed into larger clumps toward the nuclear envelope (Fig. 1A) (15-24 min) and collapsed eventually in a few nuclear fragments. In other cells (Fig. $1 B$ ), the formation of many small
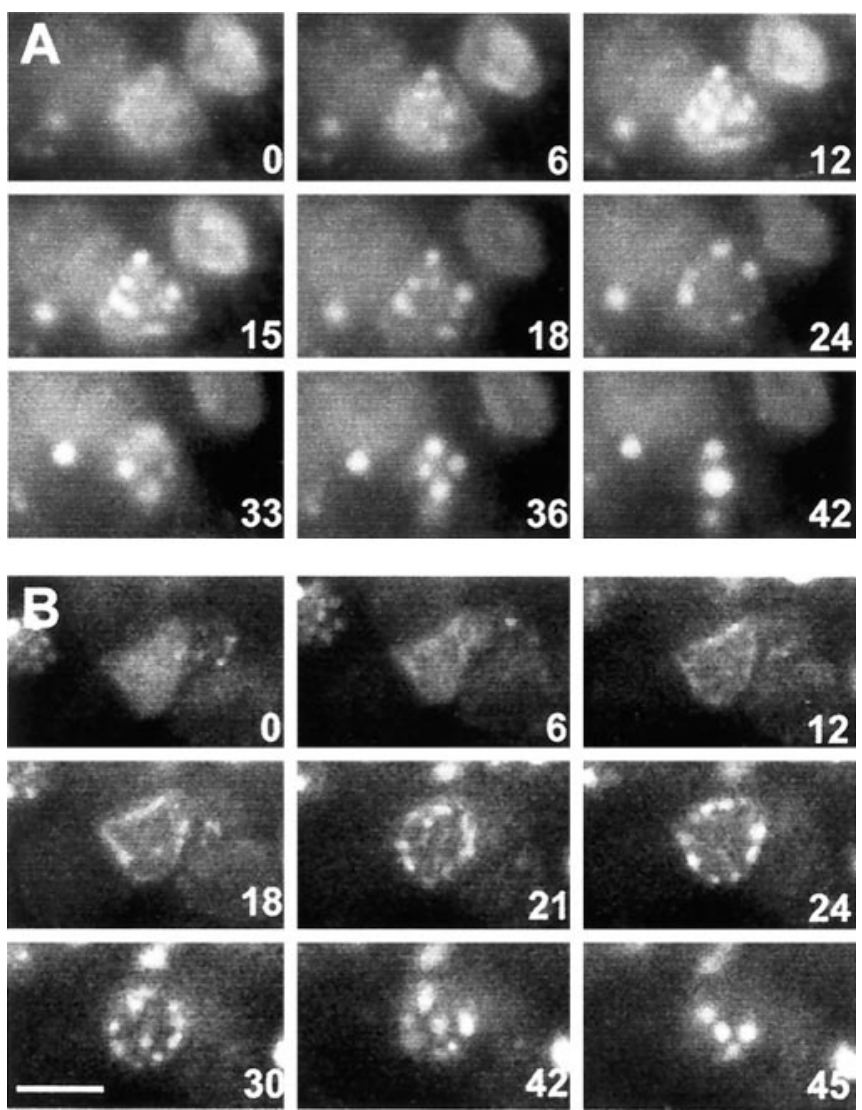

Figure 1. Two examples of P1 rat retinal ganglion cells undergoing apoptosis. Retinal ganglion cells were retrogradely labeled with DiY in vivo. The number on each panel corresponds to the minutes since the beginning of the sequence. $A$, The first detectable sign of apoptosis in most cells is the formation of many small clumps within the nucleus. $B$, In same cases the first morphological sign of apoptosis is a thin rim of condensed chromatin at the outer edge of the nucleus. Scale bars, $10 \mu \mathrm{m}$.

nuclear clumps was not evident. Rather, the first detectable sign of pyknosis was the appearance of a thin rim of denser chromatin close to the nuclear envelope (Fig. 1B) (12-18 min). Chromatin later condensed in large clumps along the nuclear envelope (Fig. $1 B)(21-24 \mathrm{~min})$ and collapsed in a few dense nuclear fragments, as described above. Figure 1, $A$ and $B$, illustrates two exemplar cases, but in some cells the process of nuclear condensation and fragmentation took an intermediate form, suggesting that we had just observed variations of a common theme rather than two morphologically different modalities of pyknosis. In all cases, the total sequence of nuclear alterations had the same duration and was accompanied by rounding of the cell nucleus (Figs. $1 A, 24, B$, $30)$. The analysis of dying cells under Nomarski optics revealed that ruffling of the membrane (Fig. 2) (cell 2, 10-20 min), shrinkage of the cytoplasm (Fig. 2) (cell 2, 30-50 min), and cell rounding (Fig. 2) (cells 1, 3) accompanied the fragmentation of the nucleus. The same sequence of morphological changes was detected when the preparation was monitored with Nomarski optics, without previous cell labeling (data not shown).

\section{Intracellular $\mathrm{Ca}^{2+}$ elevation during apoptosis}

The transition from healthy looking cells to overt pyknosis was systematically associated with a rise in intracellular $\mathrm{Ca}^{2+}$, which we detected by loading retinal cells with the cell-permeable, low-affinity calcium indicator Fluo-3-AM. On excitation with 448 nm light, Fluo-3-AM emits with a peak around $530 \mathrm{~nm}$ when bound to $\mathrm{Ca}^{2+}$. Given its low $\mathrm{Ca}^{2+}$ affinity, Fluo-3-AM does not emit a detectable amount of fluorescence at the physiological 


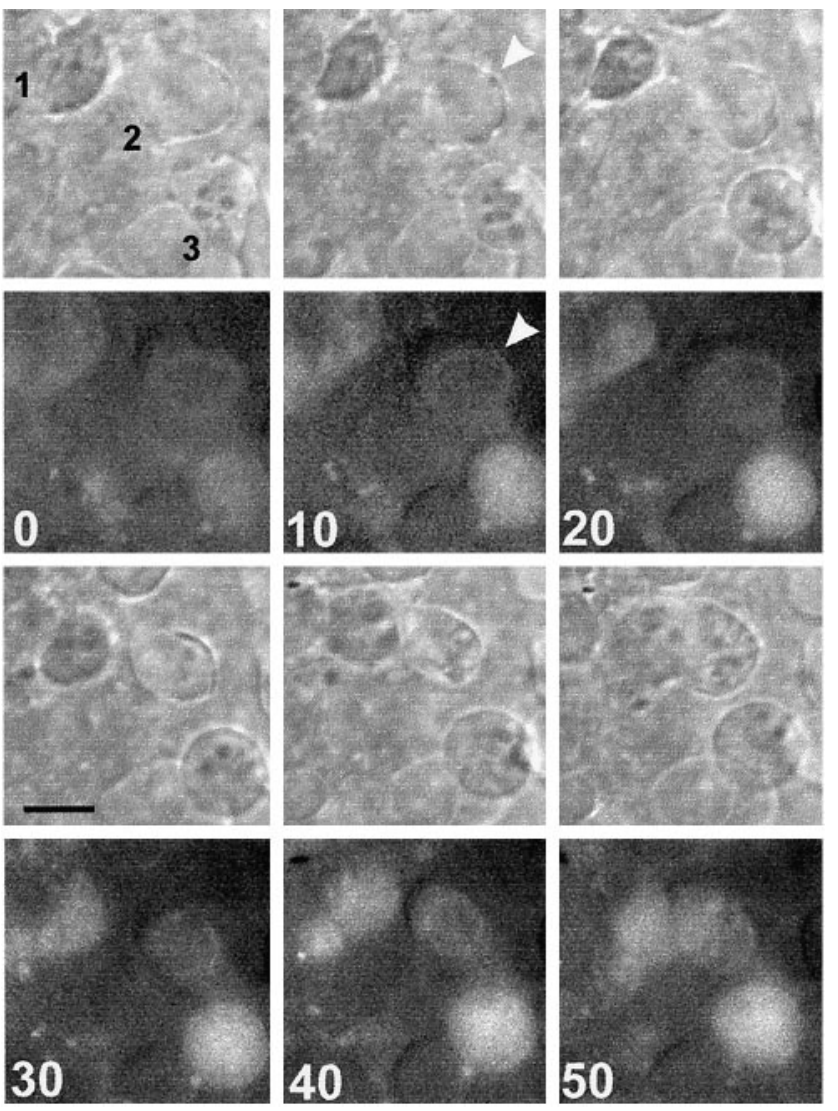

Figure 2. The sequence of morphological changes associated with cell death is accompanied by a steady increase of intracellular $\mathrm{Ca}^{2+}$ levels. A Nomarski view (top to bottom, rows 1 and 3 ) and the Fluo-3 fluorescence emission (rows 2 and 4 ) of the same field are shown at $10 \mathrm{~min}$ intervals. The minutes since the beginning of the sequence are indicated by the numbers on each bottom panel. Three dying cells can be seen in the sequence (black numbers in the Nomarski view at time 0). Cell 2 can be followed since the early stages of nuclear and cytoplasmic changes associated with death, whereas cells 1 and 3 are already in an advanced phase at time 0 . Membrane blebbing is visible for cell 2 at 10 (arrowhead) and $20 \mathrm{~min}$; nuclear fragmentation is visible at $50 \mathrm{~min}$. Nuclear fragments are already visible in cell 3 at time 0 and in cell 1 at $30 \mathrm{~min}$. Cells 1 and 3 clearly exemplify cell rounding as death proceeds. When viewed under epifluorescence, all three cells show a progressive increase in Fluo-3 emission throughout the interval illustrated. The first detectable Fluo-3 signal in cell 2 is observed at the time when membrane blebbing appears. Scale bar, $10 \mu \mathrm{m}$.

calcium levels found in normal RGCs. However, we found that as soon as a Fluo-3 signal became detectable, this was invariably associated with a newly appearing dying cell.

The overlap in the emission spectra of Fluo-3 and DiY did not allow coupling of $\mathrm{Ca}^{2+}$ imaging with DiY nuclear imaging of RGCs. Thus, we switched to parallel acquisitions of Fluo-3 and Nomarski images of the same field over time (Fig. 2). Because the low Fluo-3 signals required long exposure times $(0.5-1 \mathrm{sec})$, we reduced the frequency of acquisition to one every $10 \mathrm{~min}$ to eliminate the risk of photo damage. Three examples of the increase in intracellular $\mathrm{Ca}^{2+}$ levels associated with apoptosis, as detected by the corresponding increase in Fluo-3 emission, are shown in Figure 2, together with the corresponding Nomarski views. When viewed under epifluorescence, all three cells show a progressive increase in Fluo-3 emission throughout the interval illustrated, but only cell 2 can be followed from the early stages of morphological changes associated with death. The first detectable Fluo-3 signal in cell 2 is observed in correspondence to membrane blebbing (10 $\mathrm{min})$. The time course of Fluo-3 fluorescence increase in the same three cells is shown in Figure $3 A$, whereas

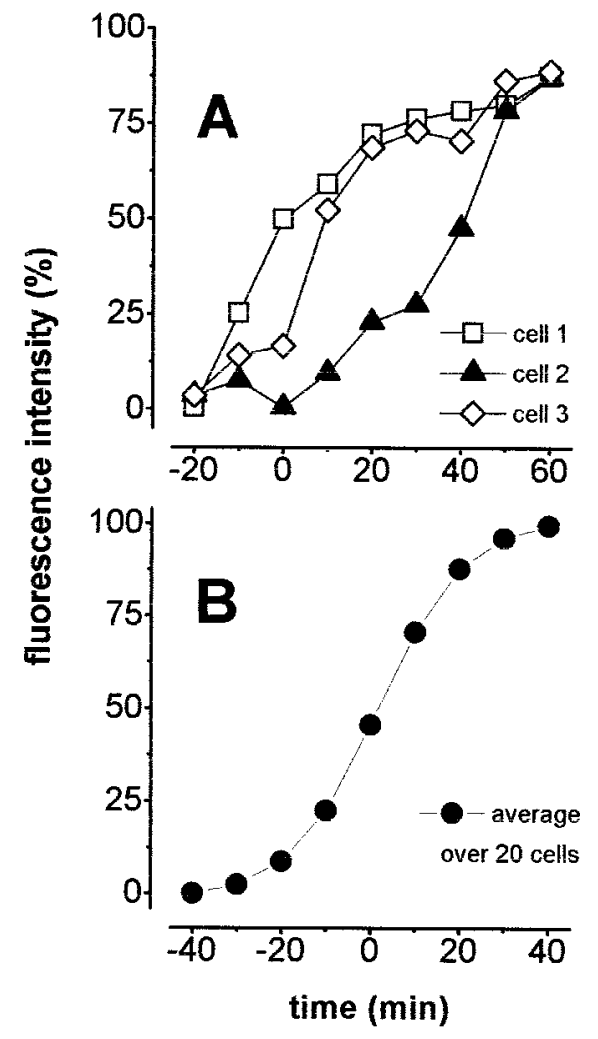

Figure 3. Time course of Fluo-3 fluorescence increase in dying cells. Fluorescence values were expressed as percentage of their maximum emission. $A$, Time course of Fluo-3 emission increase for the cells indicated in Figure 2. B, Time course of Fluo-3 emission increase averaged over 20 cells recorded in seven separate experiments. Error bars represent SDs. The curves corresponding to different cells were aligned in such a way as to superimpose the point of $50 \%$ relative fluorescence. All cells take $\sim 40 \pm 10$ min to rise from $10 \%$ to $90 \%$ of the maximal fluorescence intensity.

Figure $3 B$ illustrates the signal averaged over 20 cells. Fluo-3 emission gradually increases, taking $40 \pm 10 \mathrm{~min}$ to rise from 10 to $90 \%$ of the maximal fluorescence intensity.

\section{Pyknotic RGCs are mobile}

Observation of dying cells in the living retina, no matter whether they were detected by means of the nuclear dye DiY, under Nomarski optics, or by Fluo-3 signaling, made it obvious that pyknotic RGCs move across the retina. Examples are illustrated in Figure $4 A, B$. Mobile apoptotic RGCs were seen in all of the 54 imaging sessions that we performed, adding up to $>80 \%$ of the pyknotic RGCs that we observed in time lapse. The degree of cell movement varied greatly, and ranged from rotations without substantial tangential displacement to movements over the length of several cell diameters within a few minutes. The cells that showed conspicuous tangential movement varied their speed and direction from frame to frame, so that their movement appeared erratic, and sudden spurts alternated with periods of quiescence and unpredictable changes in direction (Fig. 4B). Different pyknotic cells monitored within the same retinal patch did not show any coherent motion, moving independently, one from the other (Fig. 4B). The only systematic feature that we could identify was that almost all pyknotic cells eventually left the RGC layer, moving away from the retina, which we detected by losing track of them in our fixed focal plane (data not shown). Assuming a linear trajectory between the positions a cell occupies in two subsequent acquisitions (i.e., every $3 \mathrm{~min}$ ), cell displacement was estimated to be between at least 20 and $50 \mu \mathrm{m} / \mathrm{hr}$, with temporary speeds reaching $200 \mu \mathrm{m} / \mathrm{hr}(10 \mu \mathrm{m}$ in $3 \mathrm{~min})$ but most frequently being 

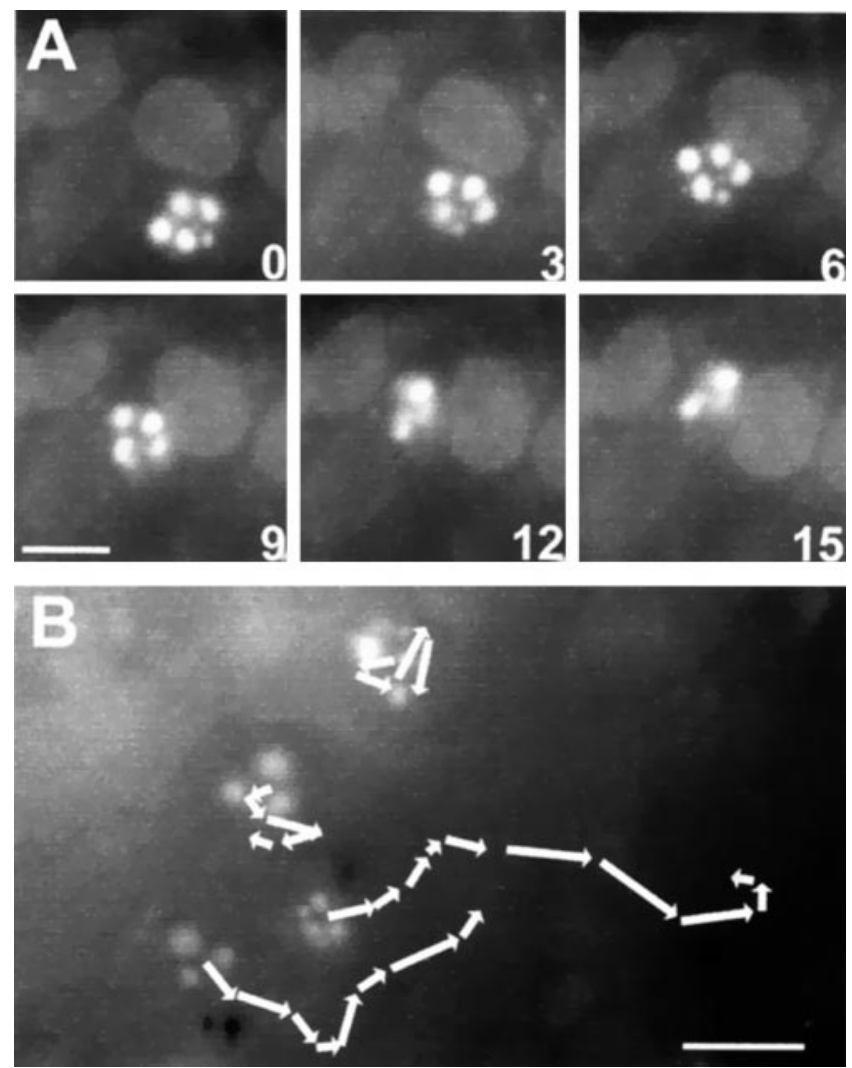

Figure 4. Erratic movement of apoptotic retinal ganglion cells. $A$, While neighboring cells are stationary, an apoptotic cells squeezes in between. The number on each panel corresponds to the minutes since the beginning of the sequence. $B$, Apoptotic cells move erratically and independently of one another. Arrows illustrate the trajectories of four cells in the same field followed for $30 \mathrm{~min}$. Scale bars, $10 \mu \mathrm{m}$.

within 10-90 $\mu \mathrm{m} / \mathrm{hr}$. Of the 15 DiY-labeled RGCs that we could follow throughout the full execution of nuclear fragmentation, 12 became mobile toward the end of the period of observation. The transition to a mobile status was roughly coincidental with the termination of the apoptotic process, with considerable variations. Dying cells started to move between 27 and 57 min after the first nuclear alterations were detected, with an average delay of $37 \pm 9 \mathrm{~min}$.

\section{DISCUSSION}

In these experiments we followed the dynamics of apoptosis in an intact preparation of a CNS structure, using time-lapse video microscopy. We determined the morphological progression and temporal profile of nuclear fragmentation, as well as the temporal profile of the intracellular $\mathrm{Ca}^{2+}$ increase associated with it. In addition, we showed that apoptotic neurons lose adhesive contacts and become mobile.

More than $50 \%$ of the retinal ganglion cells die within the first postnatal week in the rat retina (Potts et al., 1982; Perry et al., 1983; Crespo et al., 1985). The first days of life correspond also to a phase of vulnerability of retinal ganglion cells to axotomy, which causes RGCs to disappear within $48 \mathrm{hr}$ of optic nerve section (Perry et al., 1983). We cannot determine whether the cells we observed would normally have died in vivo or are dying as a consequence of the unavoidable axotomy caused by explantation. Naturally occurring and axotomy-induced death could represent different processes (Fagiolini et al., 1997; Shen et al., 1999; Cellerino et al., 2000). However, considerable experimental evidence indicates that the two processes, if different, are likely to converge on the same "execution" program, as indicated by the rescue of
RGCs from death promoted by bcl-2 overexpression or by protein synthesis inhibition in both naturally occurring and axotomyinduced death (Rabacchi et al., 1994; Bonfanti et al., 1996), as well as by the indistinguishable morphology of pyknotic cells in the two cases (Perry et al., 1983; Rabacchi et al., 1994). Within the pyknotic nucleus, chromatin appears collapsed in a few large clumps, betraying a massive and irreversible DNA fragmentation. These morphological features have been shown to unequivocally identify dying neurons in the rat retina, as shown by the correspondence of terminal deoxynucleotidyl transferase-mediated biotinylated dUTP nick end labeling-positive and pyknotic cells, where nuclear condensation was identified with a number of nuclear markers, including DY, which we have used here (Perry et al., 1983; Harvey and Robertson, 1992; Rabacchi et al., 1994). By time-lapse analysis we monitored the progression of nuclear alterations preceding pyknosis in single neurons of the living retina. Nuclear fragmentation started with the formation of small clumps, either throughout the nucleus, or close to the nuclear envelope, that later collapsed in a few larger chromatin clumps. With the sole exception of mini-clumps throughout the nucleus, cells corresponding to each of these stages have been observed in anatomical studies, as still-frames of this highly dynamic death process (Perry et al., 1983; Rabacchi et al., 1994). Nuclear fragmentation was completed in $\sim 40 \mathrm{~min}$, and little variation was observed from cell to cell or from experiment to experiment. This duration is in good agreement with the $\sim 30$ min cell death period reported for Caenorhabditis elegans (Ellis et al., 1991), the only other direct observation of dying cells in vivo, as well as with indirect estimates derived for dying retinal ganglion cells (GalliResta and Ensini, 1996). This tightly scheduled progression through nuclear fragmentation, which may represent the irreversible step in cell death, well fits the notion that death by apoptosis is a widespread, extremely conserved cell program (Oppenheim, 1991; Pettmann and Henderson, 1998).

A progressive increase of intracellular $\mathrm{Ca}^{2+}$ always accompanies the sequence of morphological changes associated with apoptosis, as we detected by means of the calcium indicator Fluo-3. The choice of a low-affinity indicator allowed us to image dying neurons without interfering with the death process, as opposed to what we observed in the same imaging conditions when using higher-affinity indicators, which most likely buffered the intracellular $\mathrm{Ca}^{2+}$ (data not shown). In our case, the first detectable Fluo-3 emission is associated with the first steps of the cytoplasmic and nuclear sequence of changes characteristic of apoptosis. Given the low affinity of Fluo-3, intracellular calcium levels are likely to increase even earlier than we could detect here. Indeed, a systematic analysis of the intracellular effectors of apoptosis in retinal ganglion cells reveals that $\mathrm{Ca}^{2+}$ entry is a necessary step for the death of these neurons (L. Colombaioni, unpublished observations), in line with a number of studies indicating that the elevation of intracellular $\mathrm{Ca}^{2+}$ levels is required in different models of cell death (Leist and Nicotera, 1998). The progressive rise of fluorescence emission observed once Fluo-3 emission begins indicates that intracellular $\mathrm{Ca}^{2+}$ levels continue to increase throughout the process of nuclear fragmentation and cell rounding. This may betray a causal relationship, because higher calcium levels may be required to induce the alteration of the cell nucleus and the cell cytoskeleton leading to fragmentation and cell rounding, but could also simply correspond to a general deregulation of the intracellular calcium buffering systems, as death proceeds to its end.

The increasing intracellular Fluo-3 fluorescence found in dying cells provides an additional piece of information: it indicates that the cell membrane remains sealed until the end of the pyknotic process, or higher than normal Fluo-3 levels would not be retained. Thus, even at its late stages, RGC apoptosis does not involve a specific leakage of intracellular material into the extracellular space. 
Monitoring the living retina by video microscopy made it evident that most apoptotic RGCs became mobile in the late stages of apoptosis, with temporary speeds reaching $200 \mu \mathrm{m} / \mathrm{hr}$. This movement appeared erratic, showing sudden spurts intercalated by periods of quiescence. All cells eventually left the RGC layer, moving away from the retina. This accounts for the common observation of pyknotic cells found slightly above the RGC layer in histological retinal preparations (L. Colombaioni, unpublished observations). It is presently unclear whether movement of apoptotic RGCs is active or whether it simply represents a passive random drift over the tissue. No matter how it arises, cell movement indicates that dying cells lose their normal contacts with the extracellular matrix and the surrounding cells, suggesting that the elimination of normal cell adhesion is a step in the process of neuronal apoptosis, an observation that parallels what has been described for dying non-neuronal cells (Frisch and Rouslahti, 1997; Meredith and Schwartz, 1997). The consistent observation of cell rounding toward the end of nuclear fragmentation could represent the morphological correlate of the cell loss of adhesion.

\section{REFERENCES}

Bonfanti L, Strettoi E, Chierzi S, Cenni MC, Liu XH, Martinou JC, Maffei L, Rabacchi SA (1996) Protection of retinal ganglion cells from natural and axotomy-induced cell death in neonatal transgenic mice overexpressing bcl-2. J Neurosci 16:4186-4194.

Cellerino A, Baehr M, Isenmann S (2000) Apoptosis in the developing visual system. Cell Tissue Res, in press.

Crespo D, O'Leary DDM, Cowan WM (1985) Changes in the number of optic nerve fibers during the late prenatal and postnatal development of the albino rat. Dev Brain Res 19:129-134.

Ellis RE, Yuan J, Horwitz HR (1991) Mechanisms and function of cell death. Annu Rev Cell Biol 7:663-698.

Fagiolini M, Caleo M, Strettoi E, Maffei L (1997) Axonal transport blockade in the neonatal rat optic nerve induces limited retinal ganglion cell death. J Neurosci 17:7045-7052.

Frisch S, Rouslahti E (1997) Integrins and anoikis. Curr Opin Cell Biol 7:701-706.

Galli-Resta L, Ensini M (1996) An intrinsic limit between genesis and death of individual neurons in the developing retinal ganglion cell layer. J Neurosci 16:2318-2324.
Hamburger V, Levi-Montalcini R (1949) Proliferation, differentiation and degeneration in the spinal ganglia of the chick embryo under normal and experimental conditions. J Exp Zool 111:457-502.

Harvey AR, Robertson D (1992) Time-course and extent of retinal ganglion cell death following ablation of the superior colliculus in neonatal rats. J Comp Neurol 325:83-94.

Leist M, Nicotera P (1998) Calcium and neuronal death. Rev Physiol Biochem Pharmacol 132:79-125.

Linden R, Rehen SK, Chiarini LB (1999) Apoptosis in developing retinal tissue. Prog Retin Eye Res 18:133-165.

MacManus JP, Rasquinha I, Black MA, Laferriere NB, Monette R, Walker T, Morley P (1997) Glutamate-treated rat cortical neuronal cultures die in a way different from the classical apoptosis induced by staurosporine. Exp Cell Res 233:310-320.

Meredith JJ, Schwartz M (1997) Integrins, adhesion and apoptosis. Trends Cell Biol 7:146-150.

Messam CA, Pittman RN (1998) Asynchrony and commitment to die during apoptosis. Exp Cell Res 238:389-398.

Oppenheim RW (1981) Neuronal cell death and some regressive phenomena during neurogenesis: a selective historical review and progress report. Studies in developmental neurobiology. Essays in honour of Viktor Hamburger (Cowan WM, ed), pp 74-133. New York: Oxford UP.

Oppenheim RW (1991) Cell death during development of the nervous system. Annu Rev Neurosci 14:453-501.

Perry VH, Henderson Z, Linden R (1983) Postnatal changes in retinal ganglion and optic axon populations in the pigmented rat. J Comp Neurol 219:356-368.

Pettmann B, Henderson CE (1998) Neuronal cell death. Neuron 20:633-647.

Pittman RN, Wang S, DiBenedetto AJ, Mills JC (1993) A system for characterizing cellular and molecular events in programmed neuronal cell death. J Neurosci 13:3669-3680.

Potts R, Dreher B, Bennet MR (1982) The loss of ganglion cells in the developing retina of the rat. Dev Brain Res 3:481-486.

Rabacchi SA, Bonfanti L, Liu XH, Maffei L (1994) Apoptotic cell death induced by optic nerve lesion in the neonatal rat. J Neurosci 14:5292-5301.

Schulz JB, Weller M, Moskowitz MA (1999) Caspases as treatment targets in stroke and neurodegenerative diseases. Ann Neurol 45:421-429.

Shen S, Wiemelt AP, McMorris FA, Barres BA (1999) Retinal ganglion cells lose trophic responsiveness after axotomy. Neuron 23:285-295.

Wyllie AH, Kerr JF, Currie AR (1980) Cell death: the significance of apoptosis. Int Rev Cytol 68:251-306. 